\title{
A Dual-Color Imaging System for Sugarcane Smut Fungus Sporisorium scitamineum
}

\begin{abstract}
Meixin Yan, Guangdong Province Key Laboratory of Microbial Signals and Disease Control, South China Agricultural University, Guangzhou, P. R. China, and Biotechnology Research Institute, Guangxi Academy of Agricultural Sciences, Guangxi, P. R. China; Enping Cai, Guangdong Innovative and Entrepreneurial Research Team of Sociomicrobiology Basic Science and Frontier Technology; Jianuan Zhou, Guangdong Province Key Laboratory of Microbial Signals and Disease Control, South China Agricultural University, Guangzhou, P. R. China; Changqing Chang, Guangdong Innovative and Entrepreneurial Research Team of Sociomicrobiology Basic Science and Frontier Technology, and Key Laboratory of Bio-Pesticide Innovation and Application of Guangdong Province, South China Agricultural University, Guangzhou, P. R. China; Pinggen Xi and Wankuan Shen, Guangdong Province Key Laboratory of Microbial Signals and Disease Control, South China Agricultural University, Guangzhou, P. R. China; Lingyu Li, Guangdong Innovative and Entrepreneurial Research Team of Sociomicrobiology Basic Science and Frontier Technology; and Zide Jiang, Yi Zhen Deng, and Lian-Hui Zhang, Guangdong Province Key Laboratory of Microbial Signals and Disease Control, South China Agricultural University, Guangzhou, P. R. China
\end{abstract}

\begin{abstract}
Yan, M. X., Cai, E. P., Zhou, J. N., Chang, C. Q., Xi, P. G., Shen, W. K., Li, L. Y., Jiang, Z. D., Deng, Y. Z., and Zhang, L.-H. 2016. A dual-color imaging system for sugarcane smut fungus Sporisorium scitamineum. Plant Dis. 100:2357-2362.

The life cycle of the sugarcane smut fungus Sporisorium scitamineum is a multistep process. Haploid sporidia of compatible (MAT-1 versus MAT-2) mating types fuse to generate pathogenic dikaryotic hyphae to infect the host. Within the host tissues, diploid teliospores are formed and induce a characteristic sorus that looks like a black whip. The diploid teliospores germinate to form haploid sporidia by meiosis. In order to monitor fungal development throughout the whole life cycle, we expressed the green fluorescent protein (GFP) and red fluorescent protein (RFP) in S. scitamineum MAT-1 and MAT-2 sporidia, respectively. Observation by epifluorescence microscope showed that conjugation

tube formation and sporidia fusion occurred at 4 to $8 \mathrm{~h}$, and formation of dikaryotic filaments was detected at $12 \mathrm{~h}$ after mating. The resultant teliospores, with diffused GFP and RFP, underwent meiosis as demonstrated by septated hypha with single fluorescent signal. We demonstrated that GFP- and RFP-tagged strains can be used to study the life cycle development of the fungal pathogen $S$. scitamineum, including the sexual mating and meiosis events. This dual-color imaging system would be a valuable tool for investigation of biotic and abiotic factors that might affect the fungal life cycle development and pathogenesis.
\end{abstract}

The basidiomycete fungus Sporisorium scitamineum is the causal agent of sugarcane smut disease, which is a serious threat in global sugarcane production. The main symptom of the disease is a characteristic whip-like sorus produced from the top of the cane or side shoots. The life cycle of $S$. scitamineum is similar to the corn smut fungus Ustilago maydis, and consists of three cell types (Albert and Schenck 1996; Kronstad and Leong 1990). Diploid teliospores are formed from the host tissues and are disseminated in the field mainly by wind or rain splashes, but the disease also spreads through the use of infected planting materials (Croft and Braithwaite 2006). The haploid sporidia emerge by teliospores germination and meiosis. Sporidia grow by budding, and compatible (MAT-1 versus $M A T-2$ ) sporidia fuse to develop into dikaryotic pathogenic hyphae, which infect plant canes and result in formation of diploid teliospores within the host tissues to complete the life cycle (Albert and Schenck 1996; Croft and Braithwaite 2006).

Corresponding authors: Lian-Hui Zhang and Yi Zhen Deng;

E-mails: lhzhang01@scau.edu.cn and dengyz@scau.edu.cn

M. X. Yan and E. P. Cai are co-first authors.

*The $e$-Xtra logo stands for "electronic extra" and indicates that one supplementary table and two supplementary figures are available online.

Accepted for publication 11 July 2016.

http://dx.doi.org/10.1094/PDIS-02-16-0257-SR

(C) 2016 The American Phytopathological Society
Regulation of sexual mating and filamentous growth in S. scitamineum is similar to what has been revealed in $U$. maydis, which relies on two mating-type loci, $a$ locus and $b$ locus (Fedler et al. 2009; Vollmeister et al. 2012; Wahl et al. 2010). The $a$ locus encodes components of the pheromone-receptor system, allowing recognition of sporidia of opposite mating type, and conjugation of mating tubes (Bölker et al. 1995; Gillissen et al. 1992). The resulting dikaryotic filament formation and activation of virulence is then controlled by the $b$ locus, which encodes two transcription factors, bE and bW (Kronstad and Leong 1990; Schlesinger et al. 1997; Schulz et al. 1990). The conserved mating type genes $a$ locus and $b$ locus have been detected in S. scitamineum (Taniguti et al. 2015), and the function of $b$ locus were characterized (Yan et al. 2016).

The GFP gene (Prasher et al. 1992) and its derivatives have been widely used to generate reporter systems to visualize the subcellular organelles as well as microbes, including many plant pathogens (Maor et al. 1998). In filamentous fungi, GFP has been successfully expressed in U. maydis (Spellig et al. 1996), Aspergillus nidulans (Fernández-Ábalos et al. 1998), and Magnaporthe oryzae (Kershaw et al. 1998). Utilization of GFP has enormously facilitated investigation on pathogen invasion of host plant tissue by Fusarium graminearum (Jansen et al. 2005), M. oryzae (Kankanala et al. 2007), Colletotrichum graminicola (Oliveira-Garcia and Deising 2013), and Zymoseptoria tritici (Kilaru et al. 2015b), and nuclear movement and division during spore germination in M. oryzae (VeneaultFourrey et al. 2006), Colletotrichum gloeosporioides (Nesher et al. 2008), Fusarium oxysporum (Ruiz-Roldan et al. 2010), U. maydis (Fink and Steinberg 2006), and Zymoseptoria tritici (Kilaru et al. 2015a). GFP-tagged protein was also routinely used to monitor 
translocation of regulatory proteins in pathogens Colletotrichum trifolii (Chen and Dickman 2002), Mycosphaerella graminicola (Rohel et al. 2001), and U. maydis (Doehlemann et al. 2009; Valinluck et al. 2014). Similarly, RFP derived from the wild type protein DsRed (Shaner et al. 2004) is also an ideal reporter protein for live-cell imaging and can be used for multiple labeling together with GFP for tracking two different organelles or proteins in the same living cell (Su et al. 2004). The first true monomeric red fluorescent protein named mRFP was developed by genetic modification of DsRed protein (Campbell et al. 2002) and was used to investigate microtubule dynamics in U. maydis (Straube et al. 2006), subcellular localization of UDP-galactopyranose mutase in A. nidulans (El-Ganiny et al. 2010), and effector secretion in M. oryzae (Ribot et al. 2013). Given that GFP and RFP were widely used in filamentous/pathogenic fungi as convenient tools for visualization of cell, cellular proteins, or subcellular organelles, cytosolic GFP or RFP respectively expressed in compatible sporidia of $S$. scitamineum are potentially a simple but versatile approach for studying fungal mating and interaction with host.

A previous study reported the expression of eGFP in $S$. scitamineum haploid spores (Sun et al. 2014). Aiming to develop useful visualizable model strains for further investigations on S. scitamineum mating and development under both in vitro and in vivo conditions, we expressed GFP and RFP in the opposite mating type sporidia of S. scitamineum for direct microscopic observation on critical differentiation steps during the life cycle of $S$. scitamineum. The results showed that expression of these fluorescent proteins in fungal cells enabled
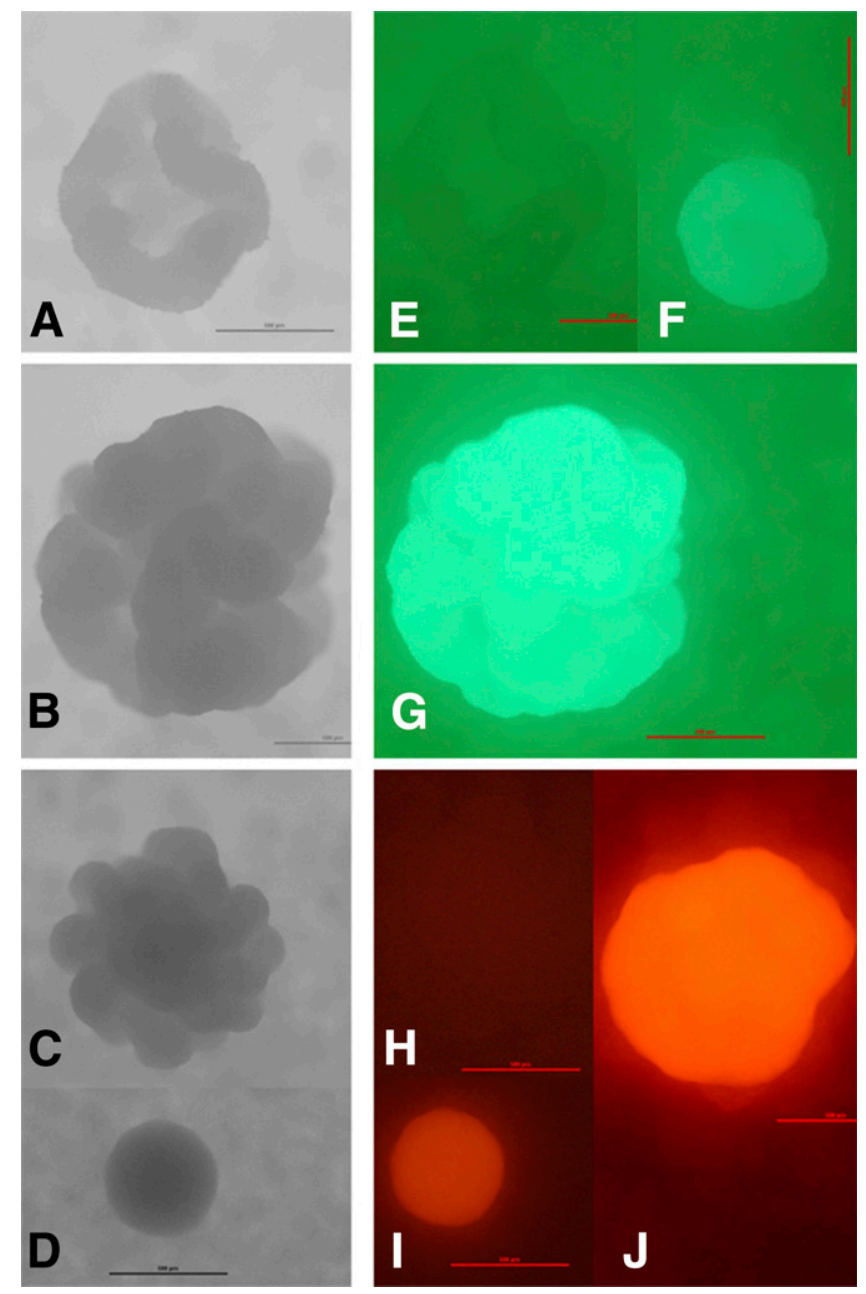

Fig. 1. Expression of GFP and RFP in Sporisorium scitamineum colonies. Wild-type MAT-1 (A) or MAT-2 (C) colonies and colonies of GFP (B) or RFP (D) tranformants in were analyzed by DIC optics. Autofluorescence was dim and negligible in the wild type $(E, H)$. Two GFP $(F, G)$ and two RFP $(I, J)$ colonies were imaged with respective filter set. Scale bar: $500 \mu \mathrm{m}$. visualization and determination of timing of the key events of fungal life cycle, such as sporidia fusion and meiosis.

\section{Materials and Methods}

Bacterial and fungal cultures. Escherichia coli strain Trans $5 \alpha$ (TransGen Biotech, CD201) was used for cloning purposes. Growth conditions and media for the cultivation of $E$. coli followed the protocols described by manufacturer (TransGen Biotech, CD201). Growth conditions as well as media for the mating type strains MAT-1 (Ss17) and MAT-2 (Ss18) of S. scitamineum have been described previously (Yan et al. 2016).

Mating and pathogenicity assays. S. scitamineum mating and pathogenicity assays were as described (Yan et al. 2016). Whip-like sorus samples were collected from an infected plant at 3 to 4 months after inoculation. Genetic transformation of $S$. scitamineum strains was as described (Sun et al. 2014). Transformants were selected on YePSA supplemented with hygromycin B $(200 \mu \mathrm{g} / \mathrm{ml})$ and cefotaxime $(300 \mu \mathrm{g} / \mathrm{ml})$, and purified on the same medium.

Plasmids used in this study. Plasmid pEX1GPD-EGFP was constructed previously (Sun et al. 2014). A 1.2-kb fragment containing mRFP and promoter $G P D$ was digested with restriction enzymes SpeI and $P a c \mathrm{I}$ and ligated into vector $\mathrm{pEX} 2$ to formed vector $\mathrm{pEX} 2-\mathrm{RFP}$. Both $e G F P$ and $m R F P$ were driven by promoter $G P D$ derived from $U$. maydis, which was as described (Sun et al. 2014). These constructs were transformed into Agrobacterium tumefaciens strain AGL1 through electroporation for genetic transformation of $S$. scitamineum.

DNA techniques. Genomic DNA from transformants and the parental wild type strains (MAT-1 and MAT-2) was isolated according to the established protocol (Lee and Taylor 1990), and was then digested by HindIII, fractionated on $1.0 \%$ agarose gel, and transferred to Nylon membranes (Roche, Cat. No. 11417240 001). The membrane was probed with about 500 bp DIG-labeled PCR-amplified fragment using Hygromycin resistance gene $h p h$ as template. Probe-hybridization was performed with PCR DIG Probe Synthesis Kit (Roche, Cat. No. 11636090 910), and detection by DIG Nucleic Acid Detection Kit (Roche, Cat. No. 11175041 910). Three nested primers were designed based on T-DNA sequences of vector $\mathrm{pEX} 2$, which is LB1: GGGTCGATGCGACGCAATCGT, LB2: ACGATG GACTCCAGAGTCCGGCCGGACCGATGGCTGTGTAGAAGT, LB3: CGATCGACAAGCTCGAGTTTCTCC, respectively. Genomic fragments were amplified using the hiTAIL-PCR combined with degenerate primers randomly, followed the protocol as described (Liu and Chen 2007). These PCR-amplified fragments were sequenced by Invitrogen Trading Co., Ltd (Shanghai).

Microscopy. For microscopic analyses, wild-type and transformed S. scitamineum cells were cultured in YePS liquid medium for $24 \mathrm{~h}$. Cells were then placed on top of a $2 \%$ agar cushion placed on glass slide, and observed and imaged using an Axioimager Z1 microscope equipped with an Axiocam MRm camera (Carl Zeiss). Standard filter sets for GFP, RFP, and DIC were used.

\section{Results}

Detection of stable epifluorescent signal in transformants. Genetic transformation of MAT-1 and MAT-2 of S. scitamineum with constructs pEX1GPD-EGFP and pEX2-RFP, respectively, yielded hygromycin-resistant fungal transformants that appeared yeast-like, with no visible anomalies. To verify T-DNA insertion and estimate the number of integrations in the individual transformants, a number of GFP and RFP transformants were chosen for Southern blot analysis. Results showed that most GFP transformants have one copy of hygromycin gene cassette (Supplementary Fig. S1, Lane 1-5 and 7-8) except for one transformant with two copies of insertion (Lane 6). Similar results were obtained with RFP transformants, in which eight out of nine transformants have one copy of hygromycin gene cassette (Lane 1 and 3-9) and the remaining one has two copies of ectopic insertion (Lane 2). To test the possibility that epifluorescent signal might be affected by T-DNA integration site, genomic sequences flanking T-DNA of GFP and RFP transformants were isolated by hiTail-PCR (Liu and Chen 2007) and sequenced. The sequencing 
results revealed that T-DNA was randomly inserted in coding or noncoding region of $S$. scitamineum genome (Supplementary Table S1).

Significant GFP signal was observed in the yeast-like colonies (Fig. 1F and G) and cells (Fig. 2I) by epifluorescent microscopy, while the colonies of wild-type untransformed MAT-1 and MAT-2 showed very weak autofluorescence (Fig. 1E). The level of epifluorescence varied among GFP transformants (Fig. 1F and G). Similarly, different levels of epifluorescence were also observed in RFP transformants (Fig. 1I and J) and cells (Fig. 2I), while autofluorescence in the wild type was weak and negligible (Fig. 1H). Next we used these two GFP- and RFP-expressed MAT-1 and MAT-2 haploid cell lines to monitor morphologic transition in mating and the following stages in S. scitamineum life cycle.

Expression of GFP and RFP during sexual mating. To examine whether GFP and RFP are suitable reporters for the infectious filament formation, we first test mating ability of transformants. MAT-1 GFP transformant was mixed with MAT-2 RFP transformant, and vise verse. All the mating mixtures, along with mixed wild-type MAT-1 and MAT-2 as control, were allowed to grow on YePSA plates in $28^{\circ} \mathrm{C}$ for 2 days. The mating results showed that all mixed transformants exhibited growth of white fluffy colonies and thus were able to mate sexually (Supplementary Fig. S2). We chose the MAT-1 strain with the strongest GFP signal, namely $M A T-1 G$, and the $M A T-2$ with the brightest RFP signal, $M A T-2 R$, for the following study.
Under epifluorescence microscope, the fungal cells were cigar-like with cytosolic GFP or RFP at the early stage of mating (Fig. 2A and I). Conjugation tubes were produced at $4 \mathrm{~h}$ post mating (Fig. $2 \mathrm{~B}$ and C, arrows), also with cytosolic GFP or RFP (Fig. 2J and K, arrows). Fusion of sporidia was observed at 4 to $8 \mathrm{~h}$ and two such events were documented under bright field (Fig. 2D and E and Fig. 2F and G) as well as under epifluorescence microscopy (Fig. 2L and M and Fig. $2 \mathrm{~N}$ and $\mathrm{O}$ ). Before fusion, GFP and RFP were separated in respective sporidia (Fig. 2D and L and Fig. 2F and N, arrows), and successful mating were indicated by merging of GFP and RFP florescence in cytoplasm of fused sporidia (Fig. 2E and $\mathrm{M}$ and Fig. 2G and O, arrows). Formation of dikaryotic filaments was detected at $12 \mathrm{~h}$ after mating (Fig. 2H and $\mathrm{P}$, arrows).

Expression of GFP and RFP in teliospores formation and germination. To analyze whether GFP and RFP can be expressed in teliospores stage, a number of mixture of GFP and RFP transformants of compatible mating types, including $M A T-1 G$ and $M A T-2 R$, were inoculated to sugarcane seedlings by injection, with mixture of untransformed MAT-1 and MAT-2 as control. After 3 months, infection symptom as black whips appeared and the induction rate of black whips, a good indicator of $S$. scitamineum pathogenicity, caused by the wild-type infection $(n=16)$ was approximately $63 \%$, while it varied from 22 to $63 \%$ for the mixed GFP- and
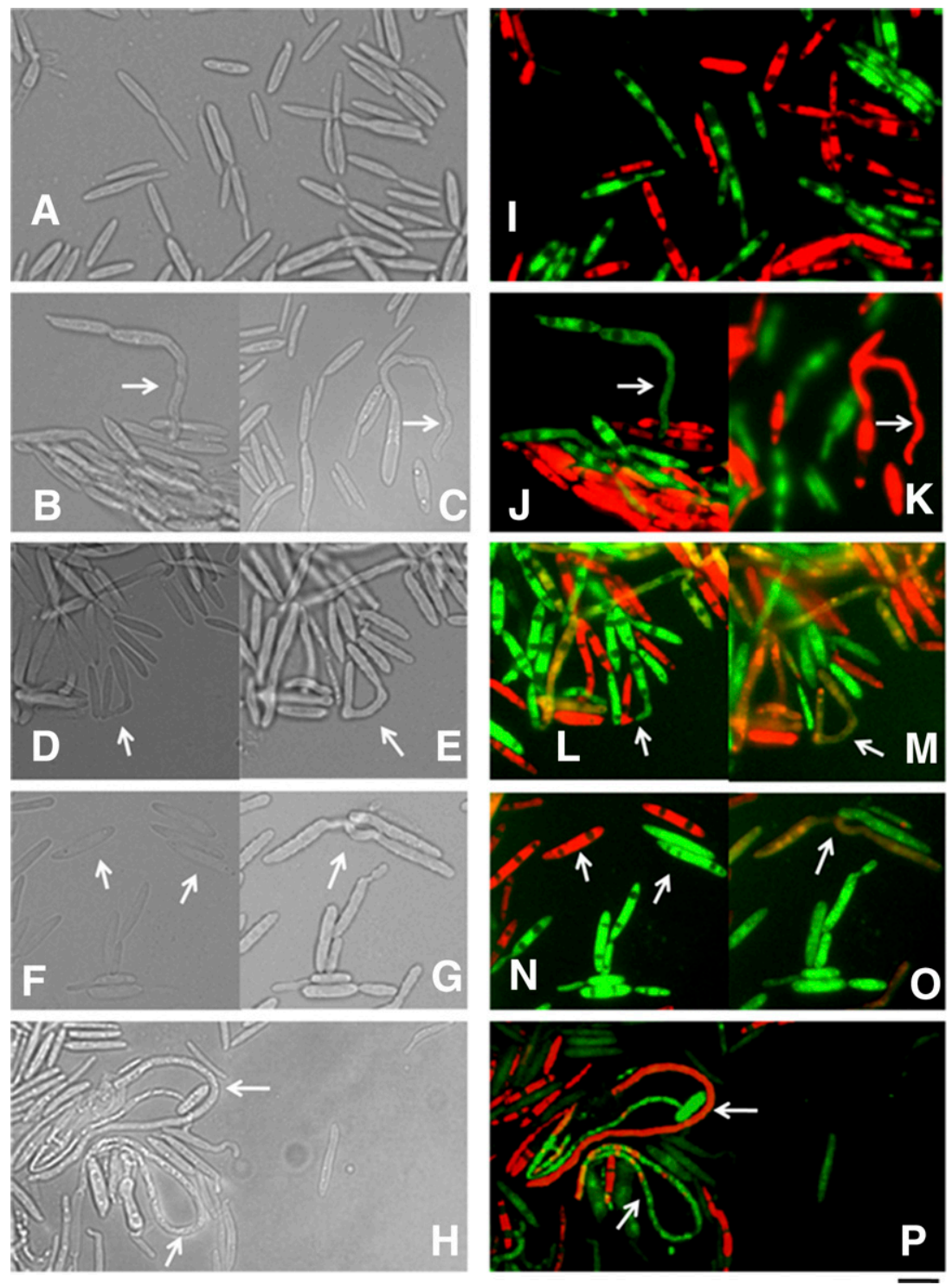

Fig. 2. Expression of GFP and RFP in Sporisorium scitamineum sexual mating. Mixture of MAT-1G and MAT-2R was inoculated on $2 \%$ agar plates. Cells were observed and imaged at $0 \mathrm{~h}(\mathbf{A}, \mathbf{I}), 4 \mathrm{~h}(\mathbf{B}, \mathbf{C}, \mathbf{D}, \mathbf{F}, \mathbf{J}, \mathrm{K}, \mathrm{L}, \mathbf{N}), 8 \mathrm{~h}(\mathbf{E}, \mathbf{G}, \mathbf{M}, \mathbf{0})$, and $12 \mathrm{~h}(\mathbf{H}, \mathbf{P})$, postinoculation. (A-H) DIC images; (I-P) epifluorescent images. Scale bar: $10 \mu \mathrm{m}$. 
RFP-transformed strains $(n=16)$. This demonstrates that $S$. scitamineum teliospore development and pathogenicity were not detrimentally affected by GFP or RFP expression (three repeats for each instance). The epifluorescent microscopy with teliospores formed by mating $M A T-1 G$ and $M A T-2 R$ showed strong GFP and RFP signal (Fig. 3A-D). Different levels of GFP and RFP were detected in teliospores sorus, and RFP signal was brighter than GFP and displayed longer duration (Fig. 3B-D). Next we followed the process of teliospore germination with this strain. At $0 \mathrm{~h}, \mathrm{GFP}$ and RFP were evenly distributed in the teliospores (Fig. 3B-D). Promycelium that consists of a short septated hypha and haploid cigar-like conidia was observed at $6 \mathrm{~h}$ (Fig. 3E), when GFP and RFP were found to coexist in same cells or distribute to different cells (Fig. 3F-H), likely due to the meiosis taking place.

\section{Discussion}

In this work, we have demonstrated the use of GFP and RFP as convenient tools for investigation on S. scitamineum life cycle development, which are critical for the fungal pathogenesis. The genomic locations of the reporter gene inserted were examined, and the effect of transgene integration on fungal growth and life cycle development were monitored to select best GFP- and RPF-tagged MAT strains as a live-cell imaging system for further investigations on the life cycle development of this important fungal pathogen. The various levels of epifluorescent signals were likely due to site effect rather than the number of integration, as the transformants harboring two copies of GFP/RFP-expression cassettes showed no stronger epifluorescence signal than those with one copy (data not shown).

Several studies reported that GFP expression leads to reduced virulence in transformed oomycetes (Dunn et al. 2013; Riedel et al. 2009) or bacteria (Sexton and Howlett 2001), likely due to the influences of insertion disruption of vital genes or the extra metabolic burden of GFP overexpression. However, our results showed that the selected transformants exhibited comparable mating ability and pathogenicity as wild-type $S$. scitamineum. This indicates that GFP and RFP expression might not necessarily interfere with the mating or virulence of $S$. scitamineum. The finding is in good agreement with the analysis of GFP effect on pathogenicity in $U$. maydis (Spellig et al. 1996) and Z. tritic (Kankanala et al. 2007; Schuster et al. 2015).

We were able to monitor the mating process of $S$. scitamineum with GFP and RFP reporter strains. Production of a long tube toward sporidia of opposite mating type was clearly seen under microscopy. Tube conjugation and fusion could be judged by diffusion of GFP and RFP signal into cytosol of opposite cells. Our results showed that using GFP and its derivative to tag compatible MAT strains could provide valuable information on critical process of fungal life cycle development. For example, by examining the relative locations of GFP and RPF fluorescence, we found that sporidia fusion, which is a good indicator of successful mating, occurred at 4 to $8 \mathrm{~h}$ post mating under the experimental conditions used in this study.
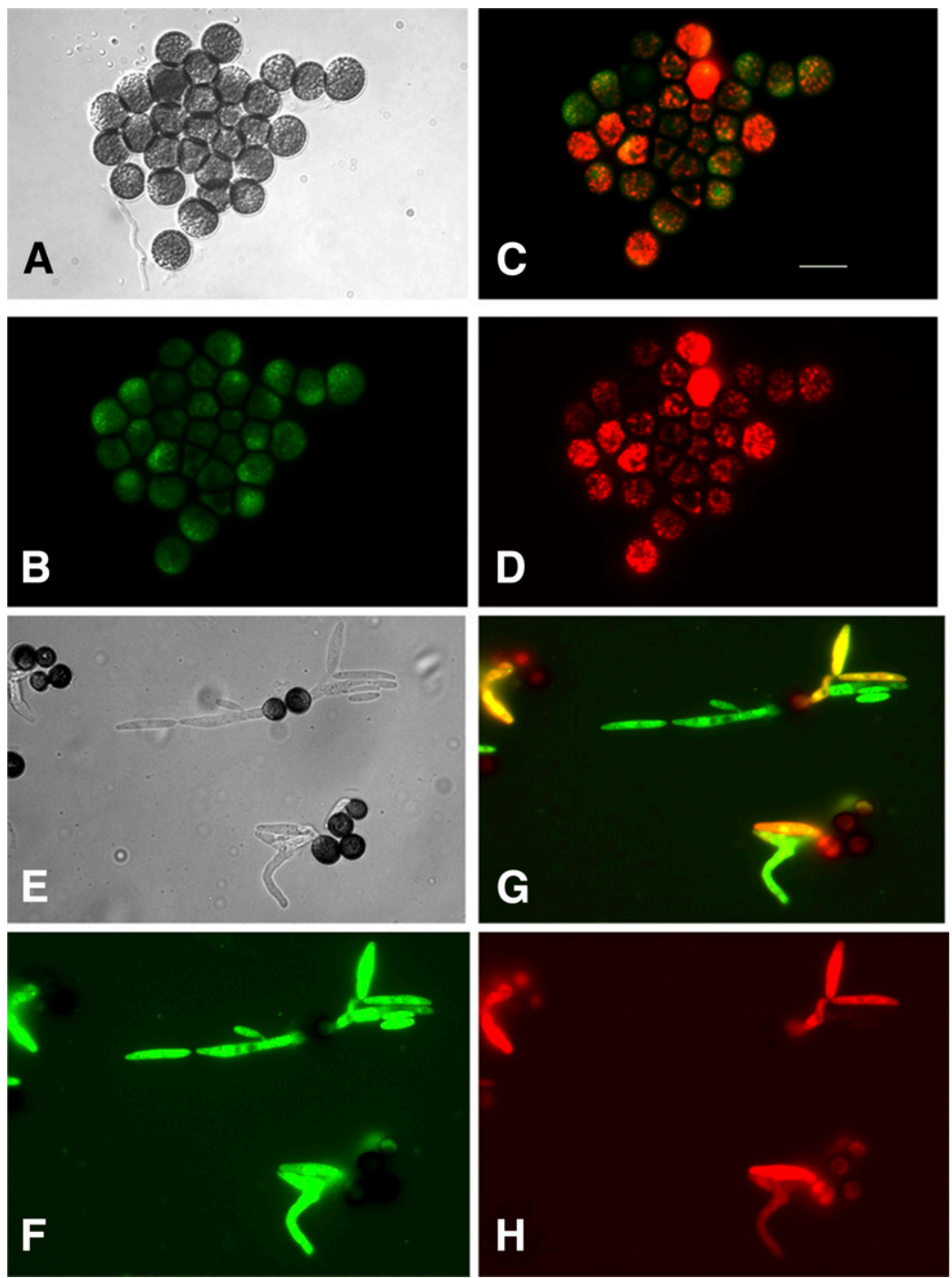

Fig. 3. Expression of GFP and RFP in Sporisorium scitamineum teliospores development. Teliospores harvested from sugar cane infected with MAT-1G $\times$ MAT-2R were incubate on $2 \%$ agar plates and imaged at $0 \mathrm{~h}$ (A-D) and $6 \mathrm{~h}(\mathrm{E}-\mathrm{H})$, postinoculation. (A, E) DIC; (B, F) GFP; (D, H) RFP; (C, G) merged images of GFP and RFP. Scale bar: $10 \mu \mathrm{m}$. 
A critical comparison of the entire infection cycle using this dualcolor imaging system, including in in planta stages, in comparison with the wild type strains would certainly benefit mycologists and plant pathologists who are working on sugarcane smut by understanding the part of the life cycle of $S$. scitamineum. However, our repeated attempts to visualize invasion of host plant tissues by transformants with GFP or RFP signal failed, due to strong autofluorescence of sugarcane tissues (data not shown). We are currently working on conditions to minimize the autofluorescence of the sugar cane tissues to facilitate observation of the fluorescence tagged fungal cells. We are also looking for alternatives, artificial surface/ materials that support dikaryotic filament growth and could be used for epifluorescent microscopy observation beyond $12 \mathrm{~h}$ post inoculation. Nevertheless, in addition to observation of fungal life cycle events, this dual-color imaging systems could also be useful for other applications, such as characterization of environmental factors that might affect the fungal life cycle and screening of inhibitors that could interfere with mating and meiosis.

In conclusion, we successfully expressed GFP and RFP in the phytopathogenic fungus $S$. scitamineum, and developed a dual-color imaging system to monitor its pathogenic life cycle. We visualized detailed structure/morphology transition and cellular fusion/ division during sexual mating and teliospore development with this system. Our results illustrate the application of such reporter system for better understanding of $S$. scitamineum life cycle development and pathogenicity. This dual-color system would be a valuable tool for further investigation of biotic and abiotic factors that might affect the pathogen life cycle and virulence.

\section{Acknowledgments}

We thank L. H. Ji and L. H. Sun for sharing the pEX2 vector. This work was funded by the National 973 Program of China (No. 2015CB150600) and the Innovative R\&D Team Program of Guangdong Province, China (No. 2013S034).

\section{Literature Cited}

Albert, H. H., and Schenck, S. 1996. PCR amplification from a homolog of the bE mating-type gene as a sensitive assay for the presence of Ustilago scitaminea DNA. Plant Dis. 80:1189-1192.

Bölker, M., Genin, S., Lehmler, C., and Kahmann, R. 1995. Genetic regulation of mating and dimorphism in Ustilago maydis. Can. J. Bot. 73(S1):320-325.

Campbell, R. E., Tour, O., Palmer, A. E., Steinbach, P. A., Baird, G. S., Zacharias, D. A., and Tsien, R. Y. 2002. A monomeric red fluorescent protein. Proc. Natl. Acad. Sci. USA 99:7877-7882.

Chen, C., and Dickman, M. B. 2002. Colletotrichum trifolii TB3 kinase, a COT1 homolog, is light inducible and becomes localized in the nucleus during hyphal elongation. Eukaryot. Cell 1:626-633

Croft, B. J., and Braithwaite, K. S. 2006. Management of an incursion of sugarcane smut in Australia. Australas. Plant Pathol. 35:113-122.

Doehlemann, G., van der Linde, K., Assmann, D., Schwammbach, D., Hof, A., Mohanty, A., Jackson, D., and Kahmann, R. 2009. Pep1, a secreted effector protein of Ustilago maydis, is required for successful invasion of plant cells. PLoS Pathog 5:e1000290.

Dunn, A., Fry, B., Lee, T., Conley, K., Balaji, V., Fry, W., McLeod, A., and Smart, C. 2013. Transformation of Phytophthora capsici with genes for green and red fluorescent protein for use in visualizing plant-pathogen interactions. Australas. Plant Pathol. 42:583-593.

El-Ganiny, A. M., Sheoran, I., Sanders, D. A., and Kaminskyj, S. G. 2010. Aspergillus nidulans UDP-glucose-4-epimerase UgeA has multiple roles in wall architecture, hyphal morphogenesis, and asexual development. Fungal Genet. Biol. 47:629-635.

Fedler, M., Luh, K. S., Stelter, K., Nieto-Jacobo, F., and Basse, C. W. 2009. The a2 mating-type locus genes lga2 and rga2 direct uniparental mitochondrial DNA (mtDNA) inheritance and constrain mtDNA recombination during sexual development of Ustilago maydis. Genetics 181:847-860.

Fernández-Ábalos, J. M., Fox, H., Pitt, C., Wells, B., and Doonan, J. H. 1998. Plant-adapted green fluorescent protein is a versatile vital reporter for gene expression, protein localization and mitosis in the filamentous fungus, Aspergillus nidulans. Mol. Microbiol. 27:121-130.

Fink, G., and Steinberg, G. 2006. Dynein-dependent motility of microtubules and nucleation sites supports polarization of the tubulin array in the fungus Ustilago maydis. Mol. Biol. Cell 17:3242-3253.

Gillissen, B., Bergemann, J., Sandmann, C., Schroeer, B., Bölker, M., and Kahmann, R. 1992. A two-component regulatory system for self/non-self recognition in Ustilago maydis. Cell 68:647-657.

Jansen, C., von Wettstein, D., Schafer, W., Kogel, K. H., Felk, A., and Maier, F. J. 2005. Infection patterns in barley and wheat spikes inoculated with wild-type and trichodiene synthase gene disrupted Fusarium graminearum. Proc. Natl. Acad. Sci. USA 102:16892-16897.

Kankanala, P., Czymmek, K., and Valent, B. 2007. Roles for rice membrane dynamics and plasmodesmata during biotrophic invasion by the blast fungus. Plant Cell 19:706-724.

Kershaw, M. J., Wakley, G., and Talbot, N. J. 1998. Complementation of the mpg 1 mutant phenotype in Magnaporthe grisea reveals functional relationships between fungal hydrophobins. EMBO J. 17:3838-3849.

Kilaru, S., Schuster, M., Latz, M., Guo, M., and Steinberg, G. 2015a. Fluorescent markers of the endocytic pathway in Zymoseptoria tritici. Fungal Genet. Biol. 79:150-157.

Kilaru, S., Schuster, M., Studholme, D., Soanes, D., Lin, C., Talbot, N. J., and Steinberg, G. 2015b. A codon-optimized green fluorescent protein for live cell imaging in Zymoseptoria tritici. Fungal Genet. Biol. 79:125-131.

Kronstad, J. W., and Leong, S. A. 1990. The $b$ mating-type locus of Ustilago maydis contains variable and constant regions. Genes Dev. 4:1384-1395.

Lee, S. and Taylor, J. 1990. Isolation of DNA from fungal mycelia and single spores. Pages 315-322 in: PCR Protocols: A Guide to Methods \& Applications. Academic Press, San Diego.

Liu, Y. G., and Chen, Y. 2007. High-efficiency thermal asymmetric interlaced PCR for amplification of unknown flanking sequences. Biotechniques 43:649-656.

Maor, R., Puyesky, M., Horwitz, B., and Sharon, A. 1998. Use of green fluorescent protein (GFP) for studying development and fungal-plant interaction in Cochliobolus heterostrophus. Mycol. Res. 102:491-496.

Nesher, I., Barhoom, S., and Sharon, A. 2008. Cell cycle and cell death are not necessary for appressorium formation and plant infection in the fungal plant pathogen Colletotrichum gloeosporioides. BMC Biol. 6:9.

Oliveira-Garcia, E., and Deising, H. B. 2013. Infection structure-specific expression of beta-1,3-glucan synthase is essential for pathogenicity of Colletotrichum graminicola and evasion of beta-glucan-triggered immunity in maize. Plant Cell 25:2356-2378.

Prasher, D. C., Eckenrode, V. K., Ward, W. W., Prendergast, F. G., and Cormier, M. J. 1992. Primary structure of the Aequorea victoria green-fluorescent protein. Gene 111:229-233.

Ribot, C., Cesari, S., Abidi, I., Chalvon, V., Bournaud, C., Vallet, J., Lebrun, M. H., Morel, J. B., and Kroj, T. 2013. The Magnaporthe oryzae effector AVR1-CO39 is translocated into rice cells independently of a fungal-derived machinery. Plant J. 74:1-12

Riedel, M., Calmin, G., Belbahri, L., Lefort, F., Gotz, M., Wagner, S., and Werres, S. 2009. Green fluorescent protein (GFP) as a reporter gene for the plant pathogenic oomycete Phytophthora ramorum. J. Eukaryot. Microbiol. 56:130-135.

Rohel, E. A., Payne, A. C., Fraaije, B. A., and Hollomon, D. W. 2001. Exploring infection of wheat and carbohydrate metabolism in Mycosphaerella graminicola transformants with differentially regulated green fluorescent protein expression. Mol. Plant-Microbe Interact. 14:156-163.

Ruiz-Roldan, M. C., Kohli, M., Roncero, M. I., Philippsen, P., Di Pietro, A., and Espeso, E. A. 2010. Nuclear dynamics during germination, conidiation, and hyphal fusion of Fusarium oxysporum. Eukaryot. Cell 9:1216-1224.

Schlesinger, R., Kahmann, R., and Kamper, J. 1997. The homeodomains of the heterodimeric $\mathrm{bE}$ and $\mathrm{bW}$ proteins of Ustilago maydis are both critical for function. Mol. Gen. Genet. 254:514-519.

Schulz, B., Banuett, F., Dahl, M., Schlesinger, R., Schafer, W., Martin, T., Herskowitz, I., and Kahmann, R. 1990. The b alleles of U. maydis, whose combinations program pathogenic development, code for polypeptides containing a homeodomain-related motif. Cell 60:295-306.

Schuster, M., Kilaru, S., Guo, M., Sommerauer, M., Lin, C., and Steinberg, G. 2015. Red fluorescent proteins for imaging Zymoseptoria tritici during invasion of wheat. Fungal Genet. Biol. 79:132-140.

Sexton, A., and Howlett, B. 2001. Green fluorescent protein as a reporter in the Brassica-Leptosphaeria maculans interaction. Physiol. Mol. Plant Pathol. 58: 13-21.

Shaner, N. C., Campbell, R. E., Steinbach, P. A., Giepmans, B. N., Palmer, A. E., and Tsien, R. Y. 2004. Improved monomeric red, orange and yellow fluorescent proteins derived from Discosoma sp. red fluorescent protein. Nat. Biotechnol. 22:1567-1572.

Spellig, T., Bottin, A., and Kahmann, R. 1996. Green fluorescent protein (GFP) as a new vital marker in the phytopathogenic fungus Ustilago maydis. Mol. Gen. Genet. 252:503-509.

Straube, A., Hause, G., Fink, G., and Steinberg, G. 2006. Conventional kinesin mediates microtubule-microtubule interactions in vivo. Mol. Biol. Cell 17:907-916.

Su, W., Li, S., Oakley, B. R., and Xiang, X. 2004. Dual-color imaging of nuclear division and mitotic spindle elongation in live cells of Aspergillus nidulans. Eukaryot. Cell 3:553-556.

Sun, L., Yan, M., Ding, Z., Liu, Y., Du, M., Xi, P., Liao, J., Ji, L., and Jiang, Z. 2014. Improved dominant selection markers and co-culturing conditions for efficient Agrobacterium tumefaciens-mediated transformation of Ustilago scitaminea. Biotechnol. Lett. 36:1309-1314.

Taniguti, L. M., Schaker, P. D., Benevenuto, J., Peters, L. P., Carvalho, G., Palhares, A., Quecine, M. C., Nunes, F. R., Kmit, M. C., Wai, A., Hausner, G., Aitken, K. S., Berkman, P. J., Fraser, J. A., Moolhuijzen, P. M., Coutinho, L. L., 
Creste, S., Vieira, M. L., Kitajima, J. P., and Monteiro-Vitorello, C. B. 2015. Complete genome sequence of Sporisorium scitamineum and biotrophic interaction transcriptome with sugarcane. PLoS One 10:e0129318.

Valinluck, M., Woraratanadharm, T., Lu, C. Y., Quintanilla, R. H., Jr., and Banuett, F. 2014. The cell end marker Tea4 regulates morphogenesis and pathogenicity in the basidiomycete fungus Ustilago maydis. Fungal Genet. Biol. 66:54-68.

Veneault-Fourrey, C., Barooah, M., Egan, M., Wakley, G., and Talbot, N. J. 2006. Autophagic fungal cell death is necessary for infection by the rice blast fungus. Science 312:580-583.
Vollmeister, E., Schipper, K., Baumann, S., Haag, C., Pohlmann, T., Stock, J., and Feldbrugge, M. 2012. Fungal development of the plant pathogen Ustilago maydis. FEMS Microbiol. Rev. 36:59-77.

Wahl, R., Zahiri, A., and Kamper, J. 2010. The Ustilago maydis b mating type locus controls hyphal proliferation and expression of secreted virulence factors in planta. Mol. Microbiol. 75:208-220.

Yan, M., Zhu, G., Lin, S., Xian, X., Chang, C., Xi, P., Shen, W., Huang, W., Cai, E., Jiang, Z., Deng, Y. Z., and Zhang, L. H. 2016. The mating-type locus b of the sugarcane smut Sporisorium scitamineum is essential for mating, filamentous growth and pathogenicity. Fungal Genet. Biol. 86:1-8. 